\title{
Effect of aerobic exercise on echocardiographic epicardial adipose tissue thickness in overweight individuals
}

\author{
Kalyana Chakravarthy \\ Bairapareddy' \\ Arun G Maiya' \\ Padma Kumar ${ }^{2}$ \\ Krishnananda Nayak ${ }^{3}$ \\ Vasudeva Guddattu ${ }^{4}$ \\ Vidya Nayak ${ }^{3}$
}

'Department of Physiotherapy, SOAHS, Manipal Academy of Higher Education, Manipal, India; ' 2 Department of Cardiology, Kasturba Medical College, Manipal Academy of Higher Education, Manipal, India; ${ }^{3}$ Department of Cardiovascular Technology, SOAHS, Manipal Academy of Higher Education, Manipal, India; ${ }^{4}$ Department of Biostatistics, Manipal Academy of Higher Education, Manipal, India
Correspondence: Kalyana Chakravarthy Bairapareddy

Department of Physiotherapy, SOAHS, Manipal Academy of Higher Education, Manipal 576104, Udupi District,

Karnataka, India

Tel +9l 8202922533

Email kalyan.reddy@manipal.edu
This article was published in the following Dove Press journal: Diabetes, Metabolic Syndrome and Obesity:Targets and Therapy

Background: Epicardial adipose tissue thickness (EATT) measured by echocardiography is a reliable indicator of visceral adipose tissue in the body and metabolic syndrome. The objective of this work was to study the effect of 12-week aerobic exercise training on echocardiographic epicardial adipose tissue thickness (EEATT), body composition and other metabolic parameters in asymptomatic overweight adults.

Methods: A total of 170 overweight and mild obese individuals were randomly allocated to 12-week aerobic exercise program (intervention group) or no supervised exercise (control group). The body fat percentage and visceral fat levels were measured by bioelectric impedance analyzer, and blood tests were conducted to measure lipids, fasting blood sugar (FBS) and high-sensitivity C-reactive protein (Hs-CRP). The participants also underwent transthoracic echocardiography to measure EATT. All the outcomes were measured after 12 weeks. Repeated measures were analyzed using ANOVA and Mann-Whitney $U$-test were used to analyze the data.

Results: EEATT values significantly reduced by $-16.24 \%$ ( $3.12 \pm 1.18$ to $2.70 \pm 1.04, p<0.05)$ following 12 weeks of aerobic exercise along with a reduction in weight by $2.96 \%(80.66 \pm 12.12$ to $78.27 \pm 12.31, p<0.05)$, body mass index by $3.11 \%(29.20 \pm 2.71$ to $28.29 \pm 2.81, p<0.05)$ and waist circumference by $2.29 \%(94.82 \pm 7.23$ to $92.64 \pm 7.33, p<0.05)$ and improved Hs-CRP values. Conclusion: The 12-week aerobic exercise was found to be effective in reducing EEATT in overweight and mild obese individuals. There was significant improvement in body composition, blood lipids, FBS, Hs-CRP and exercise capacity in intervention group compared to control group.

Keywords: aerobic exercise, epicardial adipose tissue, echocardiography, overweight

\section{Introduction}

The knowledge on adverse effects of increased visceral adipose tissue (VAT) has created new research interest in primary and secondary prevention of diseases. The overweight and obese individuals are more prone to cardiovascular disorders due to pathogenic profile of excess VAT. ${ }^{1}$ VAT is more pro-inflammatory and metabolically active compared to the subcutaneous adipose tissue (SAT) and associated with cardiovascular diseases even in nonobese population. ${ }^{2,3}$ Recently, the adipose tissue within the thoracic cavity has been found to play a greater role in development and progression of diseases. ${ }^{4}$

Epicardial adipose tissue (EAT), the fat on the surface of the myocardium and beneath the epicardium, is a known risk factor for cardiometabolic diseases in obese population..$^{5}$ There is also evidence on its pathogenic profile and clinical implications 
of increased epicardial adipose tissue thickness (EATT). ${ }^{6}$ EATT is increased in coronary artery disease (CAD) patients compared to those without CAD. The role of EAT in CAD is explained by its presence in abundance closer to coronary arteries, its ability to store excess lipids and its active role in producing inflammatory substances like C-reactive protein. ${ }^{7,8}$ EATT is increased in healthy and asymptomatic individuals with increased body mass index (BMI) across different ages, and also in lean individuals with CAD. ${ }^{9,10}$ The literature on EAT and its relationship with metabolic syndrome is mostly limited to European and Caucasian population. ${ }^{11}$ Studies on EAT in other ethnic populations, focusing on prevention of obesity-related diseases, are limited.

As the prevalence of obesity and its related complications is increasing in developing countries like India, the prevention and management of this global epidemic has gained importance recently. ${ }^{12}$ The reduction of excess body fat and visceral fat with exercise as mainstay of treatment has been demonstrated in several studies earlier. ${ }^{13,14}$ Anthropometric parameters are still widely used to monitor the effects of weight loss interventions, but the accuracy of these parameters in monitoring the changes in VAT is not good. ${ }^{15}$ The quantification of VAT using computed tomography and magnetic resonance imaging (MRI) provides accurate measurements, helping in risk stratification of obese individuals and also to monitor the effect of therapeutic interventions in primary prevention of cardiovascular diseases. ${ }^{16,17}$ It was observed that VAT or intra-abdominal fat estimated by MRI had good correlation with EATT in earlier studies. ${ }^{18}$ EATT measurement using echocardiography has been proposed to be an easy and inexpensive method for quantifying the VAT in overweight and obese population. ${ }^{19}$ The interrater and intrarater reliability of EATT measurement is found to be good, and the method has been validated with MRI. ${ }^{20}$

Echocardiographic epicardial adipose tissue thickness (EEATT) is proposed as a therapeutic target in obesity management. ${ }^{21}$ The reduction of EATT with low-calorie diet, bariatric surgery and anti-dyslipidemia drugs has been demonstrated in the previous studies. ${ }^{22-24}$ Although the reduction of VAT with aerobic and resistance exercises has been demonstrated earlier, there is scarcity of literature on the effect of exercise on EAT. ${ }^{25}$ Aerobic exercise has been found to be effective in reducing the EEATT along with a reduction in VAT in a study conducted by Kim et al in asymptomatic obese men. ${ }^{26}$ This study did not include non-exercise control group. A study conducted by Wilund et al in patients undergoing hemodialysis confirmed the role of aerobic exercise in reducing EEATT. ${ }^{27}$ The reduction in EAT was associated with an improvement in the left ventricular regional myocardial function in patients with metabolic syndrome. ${ }^{28}$ Even though there is some evidence, the magnitude of effect of aerobic exercise on EEATT has not been well characterized in a large sample-based study so far. The objective of our study was to determine the effect of aerobic exercise program on the EEATT, body weight, BMI, waist circumference (WC), blood lipids and high-sensitivity $\mathrm{C}$-reactive protein (Hs-CRP) in overweight and obese individuals.

\section{Methods}

A total of 170 overweight and mild obese Asian-Indian individuals (BMI 25.00-34.9) of age between 20 and 45 years were randomized to study group or control group after thorough screening as part of a randomized controlled trial. Institutional ethical committee approval (Manipal Academy of Higher Education, India; approval number: UEC/55/2009) was obtained prior to commencement of the study. A written informed consent was obtained from all the participants. The sample size was estimated. To detect a minimal clinically important difference of $0.8 \mathrm{~mm}$ and $\mathrm{SD}$ of $1.4 \mathrm{~mm}$ at $5 \%$ level of significance and $90 \%$ power for a two-tailed test, the sample size required in each group was 64 . But considering the high attrition rate, 85 participants were included in each group. The participants included were primary prevention population with no known diseases and those who expressed willingness to participate in the study. All the participants were recruited by verbal advertisement, mainly by conducting awareness programs in the institutions and during disease prevention camps.

\section{Randomization}

Ninety females and 80 males were allocated to one of the two groups using Sequentially Numbered Opaque Sealed Envelopes method and block randomization method. The sequence generation was not known to the primary investigator, and the treatment allocation was not known to the sonologist and medical lab technician who interpreted the blood test results.

\section{Initial screening}

A brief questionnaire was administered to know the physical activity status, and dietary and other lifestyle habits of the participants. A short version of Physical Activity Readiness Questionnaire was administered as part of the screening, and participants with known history of medical conditions were not included. ${ }^{29}$ Resting blood pressure was measured, and participants with consistent high readings were advised to go for further medical examination and were not included 
in the study. Participants who were on medications known to affect carbohydrate or lipid metabolism, or anti-inflammatory drugs, or had psychiatric disorders were excluded from the study. The exclusion criteria also included diagnosis of musculoskeletal or neurological conditions and any major nutritional deficiency disorders contraindicating the exercise test and prescription. Chronic smokers and alcoholics were also not included in the study. The eligible participants were invited for an orientation session where the explanation and clarifications about the study were given. After the randomization process, the participants were given advice on healthy weight maintenance diet, but no calorie restriction was advised. The participants who agreed to sign the informed consent were assigned to one of the two groups and underwent anthropometric assessment, body composition assessment, exercise testing, echocardiography and blood tests. The sequence of assessment and the outcome measurements are mentioned in the flowchart shown in Figure 1.

\section{Anthropometrics}

Height was measured at baseline using a wall tape calibrated to $0.1 \mathrm{~cm}$ with shoes off. Body weight was measured using an electronic weighing scale (model DS-215 series; EssaeTeraoka Limited) in light clothing without shoes to the nearest $0.1 \mathrm{~kg}$. BMI was calculated as body weight divided by height squared in $\mathrm{kg} / \mathrm{m}^{2}$. WC was measured in centimeters between the lower rib margin and the iliac crest at the end of normal expiration using a snug inch tape while the participants were standing with their heels together. The National Institutes of Health guidelines for measurement of WC and ethnic-specific risk categorization recommended for south Asians were used for interpretation. All measurements were performed after an overnight fast.

\section{Body fat measurement}

Four-electrode bioelectric impedance analyzer (HBF-362 Omron Karada Scan) was used to measure the total body

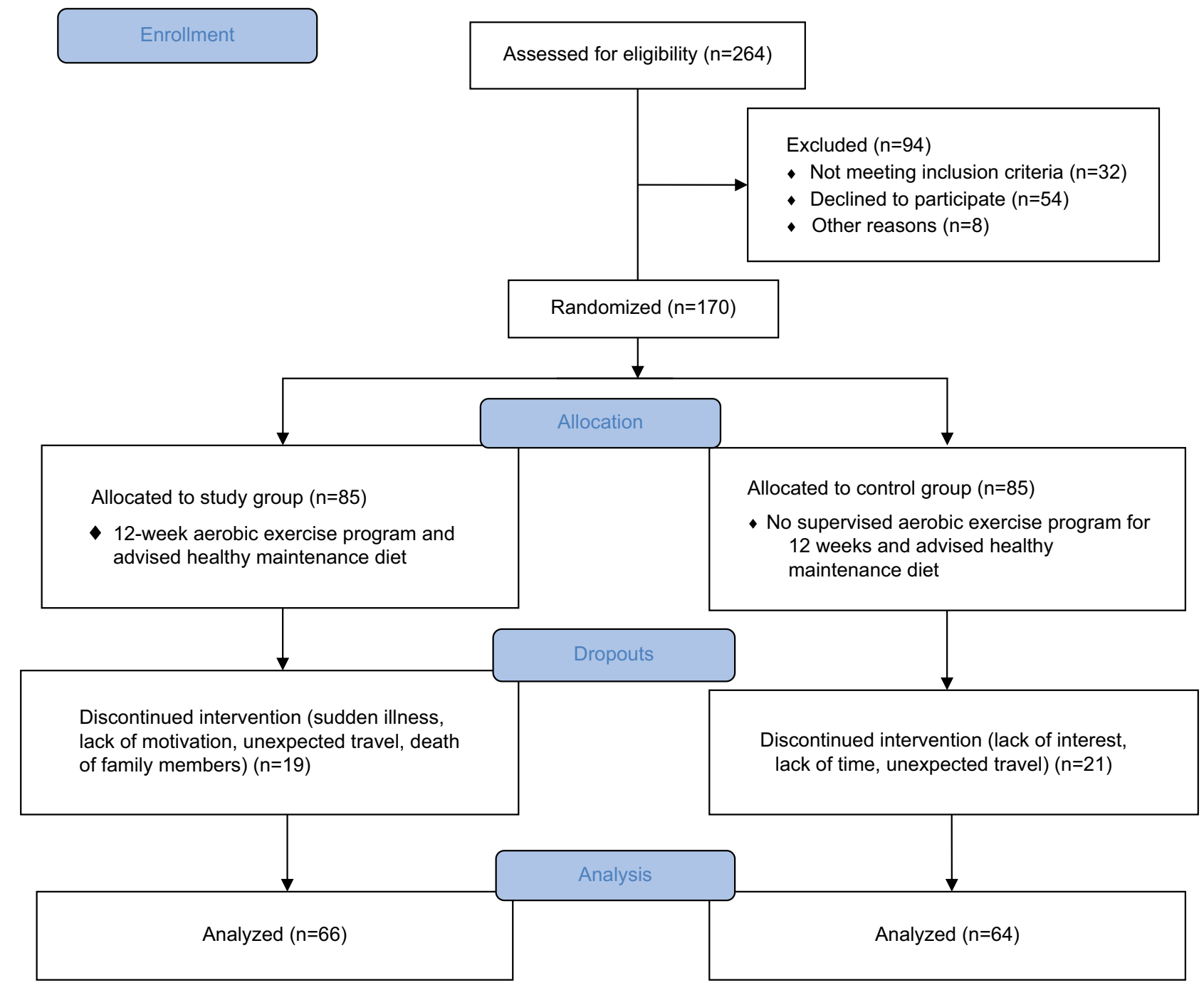

Figure I Flow diagram showing the procedure according to CONSORT guidelines. 
fat percentage and visceral fat levels. It was ensured that the participants did not consume food or water for at least 1 hour prior to the measurement. Test-retest reliability examined in the present study for total body fat percentage and visceral fat levels was found to be excellent with standard measurement errors ranging from $0.8 \%$ to $2 \%$ and $0.5 \%$ to $1.5 \%$, respectively.

\section{Blood tests}

The participants were asked to go for a blood test after overnight fasting for 10-12 hours and were also asked to refrain from consuming alcohol and exercise for 24 hours prior. Total cholesterol, low-density lipoprotein cholesterol, high-density lipoprotein cholesterol, triglycerides, fasting blood sugar (FBS) levels and Hs-CRP (immunoturbidimetric method) were measured. The blood lipid values were interpreted according to the 2002 National Cholesterol Education Program - ATP III guidelines. ${ }^{30}$

\section{EEATT measurement}

All the participants underwent transthoracic two-dimensional M-mode echocardiography (General Electric Company [GE] Vivid E echo machine). The measurements were taken in standard parasternal view in the left lateral decubitus position by an experienced sonologist, and the mean values between long- and short-axis views were recorded. The EAT was identified as the echo-free space between the outer wall of the myocardium and the visceral layer of the pericardium. The thickness was measured perpendicularly on the free wall of the right ventricle at end systole in three to ten cardiac cycles as described by Iacobellis et al. ${ }^{31}$ The images were stored in the device for accurate measurement. All the measurements were taken by a single tester, and the tester was blinded about the treatment allocation. The interobserver and intraobserver agreement (intraclass correlation coefficient 0.98 and 0.96 , respectively) for EEATT measurement was found to be excellent in a sample of 40 overweight and obese individuals participated in the present study.

\section{Assessment of physical activity and cardiorespiratory fitness}

The cardiorespiratory endurance test was conducted using treadmill testing protocol. ${ }^{32}$ A polar heart monitor (Polar FT1; Polar Electro Oy, Kempele, Finland) was used to monitor the heart rate response to the incremental exercise. The termination of the test was done as soon as the client reached the agepredicted maximum heart rate or once he/she achieved more than 17 on Borg's rate of perceived exertion scale. The duration of total exercise before the participant stopped the exercise test was recorded, and peak $\mathrm{VO}_{2}$ values were estimated.

\section{Treatment for the study group}

The American College of Sports Medicine (ACSM) guidelines were followed for the exercise prescription for the intervention group. Moderate-intensity (40\%-60\% of heart rate reserve) aerobic exercise program on treadmill was assigned to the intervention group participants. ${ }^{33}$ The continuous aerobic exercise was preceded by 5-15 minutes of warm-up session and followed by 5-10 minutes of cool-down session. Stretching exercises and low-intensity treadmill exercises were mainly the part of warm-up and cool-down sessions. The frequency of exercise was set as a minimum of three sessions in a week to all seven sessions, and each session lasted for 40-60 minutes including the warm-up and cool-down sessions. The prescribed heart rate range was maintained by adjusting the speed and inclination in the treadmill. A logbook was maintained to track the duration, intensity and frequency of the exercise sessions done by each participant. The adherence to the exercise program was calculated for each participant, and average for all participants was calculated. The investigators also conducted several counseling sessions to motivate the participants and improve the adherence to the exercise program. The total exercise minutes and energy expenditure in terms of MET minutes per week were calculated.

\section{Attrition}

The participants who missed more than three follow-up appointments or were not exercising for a minimum of three sessions in a week were contacted via telephone. Thorough attempts were made by the investigators to contact the participants and to improve adherence to the prescribed exercise program. The participants who missed more than four exercise sessions in a week and who did not complete a minimum of 30 sessions in 12-week duration were considered as dropouts. The participants in the control group who did not respond to the phone calls made to monitor physical activity levels and who did not appear for assessment and investigations after 12 weeks were considered as dropouts. The number of dropouts in both the groups was 40 , and a total of 130 participants ( 71 females and 59 males) were included for the data analysis. The overall compliance to the exercise prescription was $\sim 86 \%$ in this study.

\section{Treatment for participants in control group}

Participants in the control group were asked to maintain their current level of activity during the 12-week study period. The activity $\log$ s were given to all the participants 
and were monitored every week either by telephone or personal contact.

\section{Statistical analysis}

The descriptive statistics were used for calculating the mean/ median of demographics of the participants in both the groups. Independent $t$-test was used to compare the baseline parameters between the intervention and control groups. Shapiro-Wilk test was conducted to examine whether data met assumptions of normality of distribution, and Mauchly's test was used to examine assumptions of sphericity for repeated measures. The data met assumptions of normality and sphericity, and thus, a repeated measures ANOVA was used to compare the changes in EATT, anthropometrics and FBS and lipid levels at the baseline and after 12 weeks between the two groups. Post hoc pairwise comparisons with a Bonferroni correction were used for significant effects by "time", "group" or "group $\times$ time". The data did not meet assumptions of normality of distribution, and thus, a MannWhitney $U$-test was used to compare the changes in Hs-CRP values at baseline and after 12 weeks of intervention between the intervention and control group. The mean percentage change from baseline to 12 weeks and Cohen's " $d$ " effect sizes were used to summarize the strength of association in study and control groups. The effect size was considered as small if $d$ was $>0.2$ and $<0.5$, medium if $d$ was $>0.5$ and $<0.8$ and large if $d$ was $>0.8$. The statistical analysis was performed using SPSS Statistics version 15 (SPSS Inc.), and $p<0.05$ was set as the limit for statistical significance.

\section{Results}

A total of 85 overweight and obese individuals in intervention group and 85 overweight and mild obese individuals in control group participated in the study. The mean age of the participants in the study was 30.99 . The attrition rate was $23.52 \%$ in both the groups, but the required sample size was achieved. The anthropometric parameters, blood lipids, fasting blood glucose and peak $\mathrm{VO}_{2}$ values were all comparable at the baseline between the intervention and control groups (Table 1).

\section{Change of EEATT, anthropometrics, lipid profile, FBS levels and peak $\mathrm{VO}_{2}$ values from baseline to post-intervention in study and control groups}

EEATT values significantly reduced by $-16.24 \%$ following 12 weeks of aerobic exercise along with a reduction in weight
Table I Comparison of baseline parameters between study group and control group

\begin{tabular}{|c|c|c|c|}
\hline Variables & $\begin{array}{l}\text { Intervention } \\
\text { group } \\
(\mathrm{n}=66) \\
\text { (mean } \pm S D)\end{array}$ & $\begin{array}{l}\text { Control group } \\
(n=64) \\
(m e a n \pm S D)\end{array}$ & $p$ value \\
\hline Weight (kg) & $80.66 \pm 12.12$ & $78.54 \pm 10.22$ & 0.22 \\
\hline EEATT $(\mathrm{mm})$ & $3.14 \pm 1.17$ & $3.08 \pm 1.21$ & 0.73 \\
\hline BMI $\left(\mathrm{kg} / \mathrm{m}^{2}\right)$ & $29.20 \pm 2.71$ & $29.01 \pm 2.42$ & 0.62 \\
\hline$W C(\mathrm{~cm})$ & $94.82 \pm 7.23$ & $94.85 \pm 6.26$ & 0.98 \\
\hline TC (mg/dL) & $|87.26 \pm 25.3|$ & $|86.58 \pm 2| .48$ & 0.85 \\
\hline $\mathrm{HDL}(\mathrm{mg} / \mathrm{dL})$ & $40.50 \pm 6.07$ & $40.69 \pm 5.67$ & 0.83 \\
\hline LDL (mg/dL) & $118.29 \pm 25.96$ & $1 \mid 6.54 \pm 28.96$ & 0.67 \\
\hline TG $(\mathrm{mg} / \mathrm{dL})$ & $106.27 \pm 26.65$ & $107.08 \pm 22.75$ & 0.83 \\
\hline FBS (mg/dL) & $96.82 \pm 7.55$ & $95.90 \pm 8.65$ & 0.46 \\
\hline $\mathrm{VO}_{2}$ peak $\left(\mathrm{mL} / \mathrm{kg} / \mathrm{m}^{2}\right)$ & $33.92 \pm 3.61$ & $33.84 \pm 3.52$ & 0.88 \\
\hline
\end{tabular}

Note: $\mathrm{VO}_{2}$ peak is a measure of aerobic capacity.

Abbreviations: EEATT, echocardiographic epicardial adipose tissue thickness; BMI, body mass index; WC, waist circumference; TC, total cholesterol; HDL, highdensity lipoprotein; LDL, low-density lipoprotein; TG, triglycerides; FBS, fasting blood sugar.

by $2.96 \%$, BMI by $3.11 \%$ and WC by $2.29 \%$. The study group also showed significant improvement in lipids and $\mathrm{VO}_{2}$ max (Table 2), whereas there were no significant changes seen in the control group. There was significant reduction of Hs-CRP values in study group as compared to control group in the present study. The median values of Hs-CRP reduced from $2.95(1.80,4.97)$ to $2.70(1.55,4.02)$ in the study group compared to a change of $2.90(1.80,4.80)$ to $2.95(1.60,5.00)$ in the control group after 12 weeks of study period.

\section{Discussion}

EATT measured by echocardiography was found to be reduced with moderate-intensity aerobic exercise program in the present study. Similar findings were shown in several pharmacological and non-pharmacological interventional studies conducted on EATT measured by echocardiography. ${ }^{23}$ It was observed from the present study that EEATT values in Asian-Indian overweight and obese population are comparatively less than other ethnic populations. ${ }^{34}$ However, the cutoff values for EEATT as an indicator of metabolic syndrome in Asian-Indian population are not known. As the reasons for lower EEATT values are not known, there is still uncertainty about the role of EEATT in CAD and metabolic syndrome in Indian population. Studies on exercise-induced changes in EEATT in obese Indian individuals would help in learning its clinical applicability in obesity management. It was found in the previous studies that the exercise-induced EEATT reduction was higher in magnitude compared to reduction in SAT 
Table 2 Comparison of anthropometrics, EEATT and aerobic capacity at baseline and 12 weeks between intervention group ( $\mathrm{n}=66$ ) and control group $(\mathrm{n}=64)$

\begin{tabular}{|c|c|c|c|c|c|c|c|}
\hline \multirow[t]{2}{*}{ Measure } & \multirow[t]{2}{*}{ Group } & \multirow{2}{*}{$\begin{array}{l}\text { Baseline, } \\
\text { mean (SD) }\end{array}$} & \multirow{2}{*}{$\begin{array}{l}\text { I } 2 \text { weeks from } \\
\text { baseline, mean (SD) }\end{array}$} & \multirow[t]{2}{*}{ Cohen's d } & \multicolumn{3}{|l|}{$P$} \\
\hline & & & & & $\mathbf{G}$ & $\mathbf{T}$ & G vs T \\
\hline \multirow[t]{3}{*}{ EEATT } & Intervention & $3.12 \pm 1.18$ & $2.70 \pm 1.04$ & 0.37 & 0.28 & $<0.005$ & $<0.005$ \\
\hline & Control & $3.08 \pm 1.21$ & $3.12 \pm 1.21$ & & & & \\
\hline & & $0.8 \mathrm{I}(-0.32$ to 0.40$)$ & $<0.005(-0.76$ to -0.86$)$ & & $F=1.15$ & $F=54.5$ & $F=91.0$ \\
\hline \multirow[t]{3}{*}{ Weight } & Intervention & $80.66 \pm 12.12$ & $78.27 \pm|2.3|$ & 0.19 & 0.92 & $<0.005$ & $<0.005$ \\
\hline & Control & $78.54 \pm 10.22$ & $78.68 \pm 10.16$ & & & & \\
\hline & & $0.22(-I .27$ to $5.5 I)$ & $0.35(-5.49$ to 1.97$)$ & & $F=0.01$ & $F=13.70$ & $F=23.31$ \\
\hline \multirow[t]{3}{*}{ BMI } & Intervention & $29.20 \pm 2.71$ & $28.29 \pm 2.81$ & 0.32 & 0.74 & 0.02 & 0.006 \\
\hline & Control & $29.01 \pm 2.42$ & $29.08 \pm 2.38$ & & & & \\
\hline & & $0.15(-0.49$ to 3.00$)$ & $0.03(-1.67$ to -0.08$)$ & & $F=0.10$ & $F=5.18$ & $F=7.59$ \\
\hline \multirow[t]{3}{*}{ WC } & Intervention & $94.82 \pm 7.23$ & $92.64 \pm 7.33$ & 0.29 & 0.45 & $<0.005$ & $<0.005$ \\
\hline & Control & $94.85 \pm 6.26$ & $94.86 \pm 6.36$ & & & & \\
\hline & & $0.67(-1.88$ to 2.91$)$ & $0.60(-4.78$ to 0.10$)$ & & $F=0.56$ & $F=102.47$ & $F=132.66$ \\
\hline \multirow[t]{3}{*}{ BFP } & Intervention & $31.99 \pm 5.27$ & $29.40 \pm 5.37$ & 0.48 & 0.34 & $<0.005$ & $<0.005$ \\
\hline & Control & $31.50 \pm 4.34$ & $31.87 \pm 4.41$ & & & & \\
\hline & & $0.76(-1.83$ to 1.35$)$ & $<0.005(-4.83$ to -1.59$)$ & & $F=4.59$ & $F=93.20$ & $F=169.14$ \\
\hline \multirow[t]{3}{*}{ VF } & Intervention & $12.85 \pm 3.84$ & $10.64 \pm 3.14$ & 0.53 & 0.45 & $<0.005$ & $<0.005$ \\
\hline & Control & $12.24 \pm 3.50$ & $|2.37 \pm 3.5|$ & & & & \\
\hline & & $0.93(-1.14$ to 1.05$)$ & $<0.005(-3.23$ to -1.07$)$ & & $F=4.08$ & $F=77.48$ & $F=124.87$ \\
\hline \multirow[t]{3}{*}{$\mathrm{VO}_{2} \max$} & Intervention & $33.74 \pm 3.38$ & $40.42 \pm 2.91$ & 1.23 & $<0.005$ & $<0.005$ & $<0.005$ \\
\hline & Control & $34.14 \pm 3.24$ & $34.59 \pm 3.65$ & & & & \\
\hline & & $0.30(-0.55$ to 1.74$)$ & $<0.005$ (5.3। to 8.37$)$ & & $F=33.65$ & $F=268.89$ & $F=165.65$ \\
\hline
\end{tabular}

Note: $\mathrm{VO}_{2}$ peak is a measure of aerobic capacity.

Abbreviations: EEATT, echocardiographic epicardial adipose tissue thickness; BMI, body mass index; WC, waist circumference; BFP, body fat percentage; VF, visceral fat.

and was similar to the amount of reduction in VAT. Most of the earlier studies were conducted in moderate-to-severe obese individuals of high-risk category. Our study was the first to investigate the exercise-induced changes in EEATT in primary prevention population.

The VAT measurement using precise and reliable methods has become an important part of interventional studies in obesity management as there is evidence on its unfavorable risk profile. The use of expensive imaging methods is limited to only few research studies and may not be practically feasible for routine clinical use. The predictive ability of VAT with EEATT measurement was found to be better than WC in an earlier study. ${ }^{35}$ Although the sensitivity and specificity of EEATT are not known, it was observed that the changes of VAT and EEATT were almost similar in magnitude in one study.

In the present study, EEATT values were reduced by $16.24 \%$ with respect to change in BMI by $3.11 \%$ and reduction in WC by $2.29 \%$ (Table 2). The mean EEATT was reduced by $0.42 \mathrm{~mm}$ with respect to reduction of mean weight by $2.39 \mathrm{~kg}$, BMI by 0.91 and WC by $2.18 \mathrm{~cm}$ after 12 weeks of exercise. A reduction of 2-3 cm of WC with significant reduction in the weight was associated with the reduction in EATT in the present study. EEATT values were reduced by $8.61 \%$ along with a reduction in BMI by $4.3 \%$ and WC by $4.2 \%$ in response to 12 -week aerobic exercise in a study conducted by Kim et al. ${ }^{26}$ The non-exercise control group and female participants were not included in this study. EEATT values were reduced by $11 \%$ after lower-intensity aerobic exercise in patients undergoing hemodialysis in a study conducted by Kenneth et al. ${ }^{27}$ The magnitude of effect on EEATT was found to be higher in our study compared to the previous study conducted by Kim et al. ${ }^{26}$ This could be due to participation of younger overweight and obese individuals in our study compared to the other studies. It can be well said that EEATT reduction occurs with aerobic exercise at a higher magnitude with minimal weight loss and minimal reduction in WC. EEATT reduction of 32\% was achieved with a BMI reduction of $19 \%$ and $\mathrm{WC}$ reduction of $23 \%$ in response to 6-month low-calorie diet in a study conducted by Iacobellis et al. ${ }^{22}$ It indicates that more weight loss is associated with higher amount of EEATT reduction. The reduction of EEATT values was observed in both females $(0.37 \mathrm{~mm})$ and males $(0.55 \mathrm{~mm})$ after 12 weeks of aerobic exercise, although the 
magnitude of effect was higher in males compared to males (Tables 3 and 4). The influence of gender on cardiovascular disease risk associated with the higher values of EEATT and the effect of exercise on this important risk factor are presently not known.

Although there is evidence on the effect of aerobic exercise on body fat and more specifically the VAT, the mechanism of its effect on regional adipose tissues such as EAT is not well known. The mechanisms related to visceral fat reduction in subjects with metabolic-related diseases were found to be different from subjects without metabolicrelated diseases. There is limited understanding about the clinical implications of VAT reduction, and the significance of reduction in quantity of VAT translating to improvement in cardiovascular disease risk. The results in our study also support the fact that VAT is used more quickly as an energy resource than subcutaneous fat during aerobic exerciseinduced weight loss. Ross and Janssen suggested that physical activity with or without weight loss was associated with a reduction in VAT. ${ }^{36}$ Although there is insufficient evidence to understand the reasons for preferential VAT reduction with aerobic exercise in obese individuals, some of the probable mechanisms explained for VAT reduction include secretion of lipolytic hormones, facilitating greater post-exercise energy expenditure and fat oxidation favoring a greater negative energy balance. ${ }^{37}$ As the EAT is an indicator of VAT, we presume that the same mechanisms could have caused the EEATT reduction in the present study.

Table 3 Comparison of the outcomes between intervention and control group at baseline and 12 weeks in females

\begin{tabular}{|c|c|c|c|c|c|c|}
\hline \multirow[t]{2}{*}{ Measure } & \multicolumn{2}{|c|}{ Intervention group $(n=37)$} & \multicolumn{2}{|c|}{ Control group $(n=34)$} & \multicolumn{2}{|l|}{ Group $\times$ Time } \\
\hline & Pre & Post & Pre & Post & $F(d f I=I, d f 2=69)$ & $p$ \\
\hline Weight (kg) & $75.79 \pm \mid 1.04$ & $73.38 \pm 11.39$ & $74.30 \pm 8.60$ & $74.50 \pm 8.73$ & 72.75 & $<0.01$ \\
\hline BMI $\left(\mathrm{kg} / \mathrm{m}^{2}\right)$ & $28.94 \pm 2.80$ & $27.95 \pm 2.90$ & $29.09 \pm 2.34$ & $29.16 \pm 2.37$ & 70.33 & 0.24 \\
\hline WC (cm) & $93.56 \pm 7.32$ & $91.13 \pm 7.38$ & $93.62 \pm 6.70$ & $93.61 \pm 6.86$ & 88.12 & $<0.01$ \\
\hline EEATT $(\mathrm{mm})$ & $2.95 \pm 1.09$ & $2.58 \pm 0.85$ & $2.90 \pm 1.24$ & $2.92 \pm 1.24$ & 65.11 & $<0.01$ \\
\hline BFP (\%) & $33.79 \pm 5.37$ & $31.12 \pm 5.46$ & $32.11 \pm 4.03$ & $32.45 \pm 3.99$ & II 2.87 & $<0.01$ \\
\hline VF levels & $11.80 \pm 4.09$ & $10.22 \pm 3.78$ & $12.08 \pm 3.82$ & $12.24 \pm 3.95$ & 81.61 & $<0.01$ \\
\hline FBS (mg/dL) & $97.15 \pm 6.72$ & $94.40 \pm 5.08$ & $94.62 \pm 9.43$ & $95.13 \pm 7.73$ & 16.61 & $<0.01$ \\
\hline $\mathrm{TC}(\mathrm{mg} / \mathrm{dL})$ & $186.57 \pm 27.59$ & $|8| .84 \pm 26.45$ & $188.48 \pm 23.54$ & $186.82 \pm 19.88$ & 5.88 & $<0.01$ \\
\hline $\mathrm{HDL}(\mathrm{mg} / \mathrm{dL})$ & $40.46 \pm 6.24$ & $42.51 \pm 5.80$ & $42.00 \pm 5.68$ & $4 I .65 \pm 5.71$ & 40.36 & $<0.01$ \\
\hline LDL (mg/dL) & $118.26 \pm 24.44$ & $113.35 \pm 24.35$ & || $3.55 \pm 28.4 \mid$ & || $3.73 \pm 27.5 \mid$ & 27.15 & $<0.01$ \\
\hline TG (mg/dL) & $104.42 \pm 25.97$ & $95.84 \pm 25.15$ & $104.48 \pm 25.44$ & $106.04 \pm 25.24$ & 47.43 & $<0.01$ \\
\hline $\mathrm{VO}_{2}$ peak $(\mathrm{mL} / \mathrm{kg} / \mathrm{min})$ & $32.36 \pm 3.30$ & $39.44 \pm 2.56$ & $33.31 \pm 2.64$ & $33.54 \pm 2.81$ & 173.32 & $<0.01$ \\
\hline
\end{tabular}

Note: $\mathrm{VO}_{2}$ peak is a measure of aerobic capacity.

Abbreviations: BMI, body mass index; WC, waist circumference; EEATT, echocardiographic epicardial adipose tissue thickness; BFP, body fat percentage; VF, visceral fat; FBS, fasting blood sugar; TC, total cholesterol; HDL, high-density lipoprotein; LDL, low-density lipoprotein; TG, triglycerides.

Table 4 Comparison of the outcomes between intervention and control group at baseline and 12 weeks in males

\begin{tabular}{|c|c|c|c|c|c|c|}
\hline \multirow[t]{2}{*}{ Measure } & \multicolumn{2}{|c|}{ Intervention group $(n=29)$} & \multicolumn{2}{|c|}{ Control group $(n=30)$} & \multicolumn{2}{|l|}{ Group $\times$ Time } \\
\hline & Pre & Post & Pre & Post & $F(d f I=I, d f 2=57)$ & $p$ \\
\hline Weight (kg) & $86.13 \pm 11.00$ & $83.78 \pm 11.02$ & $83.31 \pm 9.85$ & $83.40 \pm 9.67$ & 46.80 & $<0.01$ \\
\hline BMI $\left(\mathrm{kg} / \mathrm{m}^{2}\right)$ & $29.50 \pm 2.60$ & $28.67 \pm 2.68$ & $28.92 \pm 2.54$ & $28.99 \pm 2.42$ & 50.21 & 0.24 \\
\hline WC (cm) & $96.25 \pm 6.95$ & $94.34 \pm 6.98$ & $96.23 \pm 5.47$ & $96.26 \pm 5.49$ & 55.20 & $<0.01$ \\
\hline EEATT $(\mathrm{mm})$ & $3.36 \pm 1.24$ & $2.8 \mathrm{I} \pm 0.90$ & $3.27 \pm 1.16$ & $3.29 \pm 1.16$ & 49.88 & $<0.01$ \\
\hline BFP (\%) & $29.96 \pm 4.41$ & $27.46 \pm 4.60$ & $30.8 I \pm 4.6 I$ & $31.23 \pm 4.82$ & 64.14 & $<0.01$ \\
\hline VF levels & $14.05 \pm 3.19$ & $11.72 \pm 2.61$ & $12.42 \pm 3.15$ & $12.52 \pm 2.97$ & 61.82 & $<0.01$ \\
\hline FBS (mg/dL) & $96.45 \pm 8.47$ & $93.20 \pm 6.66$ & $97.35 \pm 7.53$ & $96.92 \pm 7.73$ & 10.55 & $<0.01$ \\
\hline TC (mg/dL) & $188.02 \pm 22.80$ & $183.00 \pm 21.98$ & $184.42 \pm 18.96$ & $185.97 \pm 17.52$ & 24.06 & $<0.01$ \\
\hline $\mathrm{HDL}(\mathrm{mg} / \mathrm{dL})$ & $39.05 \pm 5.68$ & $40.40 \pm 4.68$ & $38.47 \pm 5.36$ & $38.75 \pm 4.93$ & 25.65 & $<0.01$ \\
\hline LDL (mg/dL) & $118.32 \pm 27.89$ & $113.20 \pm 27.23$ & $119.90 \pm 29.56$ & $121.17 \pm 29.06$ & 40.53 & $<0.01$ \\
\hline TG (mg/dL) & $108.35 \pm 27.58$ & $103.25 \pm 26.68$ & $110.00 \pm 19.18$ & $110.65 \pm 19.86$ & 21.44 & $<0.01$ \\
\hline $\mathrm{VO}_{2}$ peak $(\mathrm{mL} / \mathrm{kg} / \mathrm{min})$ & $35.30 \pm 2.78$ & $41.52 \pm 2.91$ & $35.08 \pm 3.62$ & $35.80 \pm 4.13$ & $|30.5|$ & $<0.01$ \\
\hline
\end{tabular}

Note: $\mathrm{VO}_{2}$ peak is a measure of aerobic capacity.

Abbreviations: BMI, body mass index; WC, waist circumference; EEATT, echocardiographic epicardial adipose tissue thickness; BFP, body fat percentage; VF, visceral fat; FBS, fasting blood sugar; TC, total cholesterol; HDL, high-density lipoprotein; LDL low-density lipoprotein; TG, triglycerides. 
EEATT values were found to be higher in obese population with dyslipidemia in various ethnic groups. Twelve-week aerobic exercise was effective in improving the metabolic parameters associated with or without significant loss in the present study. We found a significant improvement in all the metabolic parameters along with a reduction in the body fat percentage. The reduction in blood sugar levels and improvement in dyslipidemia (Table 5) were associated with a reduction in EEATT values. It implies that exercise causes physiological changes in the body leading to visceral fat reduction and further improving the cardiometabolic risk profile. The evaluation of body fat and visceral fat levels with bio impedance analysis is a more reliable method to assess body composition compared to other anthropometric measures. ${ }^{38}$ There was a significant improvement in visceral fat levels and body fat percentage after 12 weeks of aerobic exercise in the present study. The changes in Hs-CRP values were not statistically significant in intervention group compared to the control group. These findings agreed with the previous findings that only longer-duration exercise (more than 6 months) causes significant reduction in Hs-CRP values.

The relationship between EATT and aerobic capacity was first studied by Kim et al. The obese individuals with higher EEATT values were found to have lower aerobic capacity tested by a bicycle ergometer. ${ }^{39}$ The mechanism by which EEATT influences the aerobic capacity is not known. The moderate-intensity exercise of minimum 12 weeks causes physiological changes in the body leading to an improvement in the aerobic capacity. ${ }^{40,41}$ We also found that there is a significant improvement in aerobic capacity which is associated with reduced EEATT values and improvement in metabolic parameters.

To the best of our knowledge, this was the first randomized controlled trial to study the efficacy of aerobic exercise on EEATT in both men and women, also in Asian-Indian population. The limitation of the study is that the normalweight individuals with increased values of EATT were not included in the study. Future studies may focus on determining the effect of aerobic exercise on EATT in normal-weight individuals. The dose-response relation between aerobic exercise and EEATT can be further studied. The nutraceuticals are also known to influence the blood cholesterol levels and thus reduce the burden of atherosclerosis. ${ }^{42}$ In the present study, the food intake during the study period was not monitored which can be considered as a confounding factor. High-volume training and high-intensity resistance training induced faster visceral loss in a study conducted by Dutheil et al. ${ }^{43}$ The effect of resistance exercises and in combination with aerobic exercises with varying volumes can be studied in future. Future research should focus on studying the sensitivity and specificity of EEATT measurements in visceral fat monitoring.

\section{Conclusion}

The 12-week aerobic exercise is effective in reducing EATT measured by echocardiography. The reduction in EEATT is associated with an improvement in anthropometrics, aerobic capacity, lipid profile and Hs-CRP.

Table 5 Comparison of FBS and lipids at baseline and 12 weeks between intervention group ( $n=66$ ) and control group ( $n=64$ )

\begin{tabular}{|c|c|c|c|c|c|c|c|}
\hline \multicolumn{2}{|c|}{ Measure } & \multirow{3}{*}{$\begin{array}{l}\text { Baseline mean (SD) } \\
96.82 \pm 7.55 \\
\end{array}$} & \multirow{3}{*}{$\begin{array}{l}\begin{array}{l}\text { } 2 \text { weeks from } \\
\text { baseline, mean (SD) }\end{array} \\
93.83 \pm 5.87\end{array}$} & \multirow{3}{*}{$\begin{array}{l}\text { Cohen's d } \\
0.44\end{array}$} & \multicolumn{3}{|l|}{$p$} \\
\hline & & & & & \multirow{2}{*}{$\begin{array}{l}\mathbf{G} \\
0.16\end{array}$} & \multirow{2}{*}{$\begin{array}{l}\mathbf{T} \\
<0.005\end{array}$} & \multirow{2}{*}{$\begin{array}{l}\text { G vs T } \\
<0.005\end{array}$} \\
\hline FG & Intervention & & & & & & \\
\hline & Control & $95.90 \pm 8.65$ & $95.97 \pm 7.55$ & & & & \\
\hline & & $0.46(-1.54$ to 3.37$)$ & $0.01(-6.32$ to -1.83$)$ & & $F=1.99$ & $F=19.45$ & $F=37.28$ \\
\hline \multirow[t]{3}{*}{ TC } & Intervention & $|87.25 \pm 25.3|$ & $|82.38 \pm 24.3|$ & 0.19 & 0.53 & $<0.005$ & $<0.005$ \\
\hline & Control & $|86.57 \pm 2| .48$ & $186.42 \pm 18.70$ & & & & \\
\hline & & $0.13(-2.15$ to 15.28$)$ & $0.74(-9.48$ to $6.8 \mathrm{I})$ & & $F=0.38$ & $F=36.88$ & $F=45.84$ \\
\hline \multirow[t]{3}{*}{$\mathrm{HDL}$} & Intervention & $39.80 \pm 5.77$ & $41.40 \pm 5.44$ & 0.28 & 0.04 & 0.02 & 0.006 \\
\hline & Control & $41.40 \pm 5.44$ & $40.28 \pm 5.52$ & & & & \\
\hline & & $0.10(-0.39$ to 3.99$)$ & $<0.005$ (2.42 to 6.56$)$ & & $F=8.72$ & $F=40.15$ & $F=62.00$ \\
\hline \multirow[t]{3}{*}{ LDL } & Intervention & $118.29 \pm 25.96$ & $113.28 \pm 25.59$ & 0.19 & 0.62 & $<0.005$ & $<0.005$ \\
\hline & Control & $116.54 \pm 28.96$ & $117.23 \pm 28.33$ & & & & \\
\hline & & $0.93(-10.02$ to 10.96$)$ & $0.28(-15.83$ to 4.68$)$ & & $F=0.23$ & $F=32.62$ & $F=40.73$ \\
\hline \multirow[t]{3}{*}{ TG } & Intervention & $106.27 \pm 26.65$ & $99.32 \pm 25.99$ & 0.26 & 0.19 & $<0.005$ & $<0.005$ \\
\hline & Control & $107.08 \pm 22.75$ & $108.09 \pm 22.66$ & & & & \\
\hline & & $\mathrm{I} .00(-9.4 \mathrm{I}$ to $9.4 \mathrm{I})$ & $0.008(-20.97$ to -3.19$)$ & & $F=1.73$ & $F=16.32$ & $F=81.74$ \\
\hline
\end{tabular}

Abbreviations: FBS, fasting blood sugar; TC, total cholesterol; HDL, high-density lipoprotein; LDL, low-density lipoprotein; TG, triglycerides. 


\section{Acknowledgments}

The authors acknowledge the contribution of lab assistants during the study period. The abstract of this paper was presented at the Twenty-Third Annual Scientific Congress, organized by Hong Kong College of Cardiology, held on 29-31 May 2015 in Hong Kong, and was published in the Journal of the Hong Kong College of Cardiology.

\section{Disclosure}

The authors report no conflicts of interest in this work.

\section{References}

1. Baker AR, Silva NF, Quinn DW, et al. Human epicardial adipose tissue expresses a pathogenic profile of adipocytokines in patients with cardiovascular disease. Cardiovasc Diabetol. 2006;5(1):1.

2. Wajchenberg BL. Subcutaneous and visceral adipose tissue: their relation to the metabolic syndrome. Endocr Rev. 2000;21(6):697-738.

3. Nakamura T, Tokunaga K, Shimomura I, et al. Contribution of visceral fat accumulation to the development of coronary artery disease in nonobese men. Atherosclerosis. 1994;107(2):239-246.

4. Matsuzawa Y, Nakamura T, Shimomura I, Kotani K. Visceral fat accumulation and cardiovascular disease. Obes Res. 1995;3(S5): 645S-647S.

5. Yerramasu A, Dey D, Venuraju S, et al. Increased volume of epicardial fat is an independent risk factor for accelerated progression of sub-clinical coronary atherosclerosis. Atherosclerosis. 2012;220(1):223-230.

6. Iacobellis G, Willens HJ. Echocardiographic epicardial fat: a review of research and clinical applications. JAm Soc Echocardiogr. 2009;22(12): 1311-1319.

7. Eroglu S, Sade LE, Yildirir A, et al. Epicardial adipose tissue thickness by echocardiography is a marker for the presence and severity of coronary artery disease. Nutr Metab Cardiovasc Dis. 2009;19(3): 211-217.

8. Ahn SG, Lim HS, Joe DY, et al. Relationship of epicardial adipose tissue by echocardiography to coronary artery disease. Heart. 2008;94(3):e7.

9. Rabkin SW. Epicardial fat: properties, function and relationship to obesity. Obes Rev. 2007;8(3):253-261.

10. Silaghi A, Piercecchi-Marti MD, Grino M, et al. Epicardial adipose tissue extent: relationship with age, body fat distribution, and coronaropathy. Obesity (Silver Spring). 2008;16(11):2424-2430.

11. Willens HJ, Gómez-Marín O, Chirinos JA, Goldberg R, Lowery MH, Iacobellis G. Comparison of epicardial and pericardial fat thickness assessed by echocardiography in African American and non-Hispanic White men: a pilot study. Ethn Dis. 2008;18(2):311-316.

12. Misra A, Pandey RM, Devi JR, Sharma R, Vikram NK, Khanna N. High prevalence of diabetes, obesity and dyslipidaemia in urban slum population in northern India. Int J Obes Relat Metab Disord. 2001;25(11):1722-1729.

13. Slentz CA, Aiken LB, Houmard JA, et al. Inactivity, exercise, and visceral fat. STRRIDE: a randomized, controlled study of exercise intensity and amount. J Appl Physiol (1985). 2005;99(4):1613-1618.

14. Ohkawara K, Tanaka S, Miyachi M, Ishikawa-Takata K, Tabata I. A dose-response relation between aerobic exercise and visceral fat reduction: systematic review of clinical trials. Int $J$ Obes (Lond). 2007;31(12):1786-1797.

15. Rankinen T, Kim SY, Pérusse L, Després JP, Bouchard C. The prediction of abdominal visceral fat level from body composition and anthropometry: ROC analysis. Int J Obes Relat Metab Disord. 1999;23(8):801-809.

16. Kaul S, Rothney MP, Peters DM, et al. Dual-energy X-ray absorptiometry for quantification of visceral fat. Obesity (Silver Spring). 2012;20(6):1313-1318.
17. Seidell JC, Bakker CJ, van der Kooy K. Imaging techniques for measuring adipose-tissue distribution - a comparison between computed tomography and 1.5-T magnetic resonance. Am J Clin Nutr. 1990;51(6):953-957.

18. Iacobellis G, Assael F, Ribaudo MC, et al. Epicardial fat from echocardiography: a new method for visceral adipose tissue prediction. Obes Res. 2003;11(2):304-310.

19. Iacobellis G. Imaging of visceral adipose tissue: an emerging diagnostic tool and therapeutic target. Curr Drug Targets Cardiovasc Hematol Disord. 2005;5(4):345-353.

20. Iacobellis G, Willens HJ, Barbaro G, Sharma AM. Threshold values of high-risk echocardiographic epicardial fat thickness. Obesity (Silver Spring). 2008;16(4):887-892.

21. Iacobellis G, Sharma AM. Epicardial adipose tissue as new cardiometabolic risk marker and potential therapeutic target in the metabolic syndrome. Curr Pharm Des. 2007;13(21):2180-2184.

22. Iacobellis G, Singh N, Wharton S, Sharma AM. Substantial changes in epicardial fat thickness after weight loss in severely obese subjects. Obesity (Silver Spring). 2008;16(7):1693-1697.

23. Willens HJ, Byers P, Chirinos JA, Labrador E, Hare JM, de Marchena E. Effects of weight loss after bariatric surgery on epicardial fat measured using echocardiography. Am J Cardiol. 2007;99(9):1242-1245.

24. Park JH, Park YS, Kim YJ, et al. Effects of statins on the epicardial fat thickness in patients with coronary artery stenosis underwent percutaneous coronary intervention: comparison of atorvastatin with simvastatin/ ezetimibe. J Cardiovasc Ultrasound. 2010;18(4):121-126.

25. Ismail I, Keating SE, Baker MK, Johnson NA. A systematic review and meta-analysis of the effect of aerobic vs. resistance exercise training on visceral fat. Obes Rev. 2012;13(1):68-91.

26. Kim MK, Tomita T, Kim MJ, Sasai H, Maeda S, Tanaka K. Aerobic exercise training reduces epicardial fat in obese men. J Appl Physiol (1985). 2009;106(1):5-11.

27. Wilund KR, Tomayko EJ, Wu PT, et al. Intradialytic exercise training reduces oxidative stress and epicardial fat: a pilot study. Nephrol Dial Transplant. 2010;25(8):2695-2701.

28. Serrano-Ferrer J, Crendal E, Walther G, et al. Effects of lifestyle intervention on left ventricular regional myocardial function in metabolic syndrome patients from the RESOLVE randomized trial. MetabolismClinical and Experimental. 2016;65(9):1350-1360.

29. Warburton DE, Jamnik VK, Bredin SSD, Gledhill N; PAR-Q+ Research Collaboration. The Physical Activity Readiness Questionnaire for Everyone (PAR-Q+) and electronic Physical Activity Readiness Medical Examination (ePARmed-X+). Health Fitness J Can. 2011;4(2):3-17.

30. Heng D, Ma S, Lee JJ, et al. Modification of the NCEP ATP III definitions of the metabolic syndrome for use in Asians identifies individuals at risk of ischemic heart disease. Atherosclerosis. 2006;186(2):367-373.

31. Iacobellis G, Willens HJ. Echocardiographic epicardial fat: a review of research and clinical applications. Journal of the American Society of Echocardiography. 2009;22(12):1311-1319.

32. Rowland TW. Aerobic exercise testing protocols. In: Rowland TW, editor. Pediatric Laboratory Exercise Testing: Clinical Guidelines. Champaign, IL: Human Kinetics; 1993:19-41.

33. American College of Sports Medicine. ACSM's Guidelines for Exercise Testing and Prescription. Baltimore, MD: Lippincott Williams \& Wilkins; 2013.

34. Xu Y, Cheng X, Hong K, Huang C, Wan L. How to interpret epicardial adipose tissue as a cause of coronary artery disease: a meta-analysis. Coron Artery Dis. 2012;23(4):227-233.

35. Natale F, Tedesco MA, Mocerino R, et al. Visceral adiposity and arterial stiffness: echocardiographic epicardial fat thickness reflects, better than waist circumference, carotid arterial stiffness in a large population of hypertensives. Eur J Echocardiogr. 2009;10(4): 549-555.

36. Ross R, Janssen I. Physical activity, total and regional obesity: doseresponse considerations. Med Sci Sports Exerc. 2001;33(6 Suppl):S521S527; discussion S528-S529. 
37. Park HS, Lee K, Greater beneficial effects of visceral fat reduction compared with subcutaneous fat reduction on parameters of the metabolic syndrome: a study of weight reduction programmes in subjects with visceral and subcutaneous obesity. Diabet Med. 2005;22(3):266-272.

38. Meleleo D, Bartolomeo N, Cassano L, et al. Evaluation of body composition with bioimpedence. A comparison between athletic and non-athletic children. Eur J Sport Sci. 2017;17(6):710-719.

39. Kim MK, Tanaka K, Kim MJ, et al. Epicardial fat tissue: relationship with cardiorespiratory fitness in men. Med Sci Sports Exerc. 2010;42(3):463-469.

40. Gaesser GA, Rich RG. Effects of high- and low-intensity exercise training on aerobic capacity and blood lipids. Med Sci Sports Exerc. 1984;16(3):269-274.
41. Wong PC, Chia MY, Tsou IY, et al. Effects of a 12-week exercise training programme on aerobic fitness, body composition, blood lipids and C-reactive protein in adolescents with obesity. Ann Acad Med Singapore. 2008;37(4):286-293.

42. Scicchitano P, Cameli M, Maiello M, et al. Nutraceuticals and dyslipidaemia: beyond the common therapeutics. J Funct Foods. 2014;6: 11-32.

43. Dutheil F, Lac G, Lesourd B, et al. Different modalities of exercise to reduce visceral fat mass and cardiovascular risk in metabolic syndrome: the RESOLVE randomized trial. Int J Cardiol. 2013;168(4): 3634-3642.
Diabetes, Metabolic Syndrome and Obesity: Targets and Therapy is an international, peer-reviewed open-access journal committed to the rapid publication of the latest laboratory and clinical findings in the fields of diabetes, metabolic syndrome and obesity research. Original research, review, case reports, hypothesis formation, expert opinion and commentaries are all considered for publication. The manuscript management system is completely online and includes a very quick and fair peer-review system, which is all easy to use. Visit http://www.dovepress.com/testimonials.php to read real quotes from published authors.

Submit your manuscript here: https://www.dovepress.com/diabetes-metabolic-syndrome-and-obesity-targets-and-therapy-journal 